\title{
Upregulation of the gene expression of CLOCK is correlated with hypoxia-inducible factor $1 \alpha$ in advanced varicose lesions
}

\author{
XIAO TANG $^{1 *}$, DAQIAO GUO ${ }^{1 *}$, CHANGPO LIN $^{1}$, ZHENYU SHI $^{1}$, RUIZHE QIAN $^{2}$, \\ WEIGUO FU ${ }^{1}$, JIANJUN LIU ${ }^{3}, \mathrm{XU} \mathrm{LI}^{3}$ and LONGHUA FAN ${ }^{3}$ \\ ${ }^{1}$ Department of Vascular Surgery, Institute of Vascular Surgery, Zhongshan Hospital, Fudan University; \\ ${ }^{2}$ Department of Physiology and Pathophysiology, Fudan University Shanghai Medical College; \\ ${ }^{3}$ Department of Vascular Surgery, Qingpu Branch of Zhongshan Hospital, Fudan University,
}

Shanghai 200032, P.R. China

Received October 19, 2014; Accepted July 21, 2015

DOI: $10.3892 / \mathrm{mmr} .2015 .4223$

\begin{abstract}
According to previous literature, venous hypoxia and the hypoxia-inducible factor (HIF) pathway may contribute to the pathogenesis of varicose veins (VVs). It is widely accepted that the circadian locomotor output cycles kaput (CLOCK) gene affects nucleotide excision repair, DNA damage checkpoints and apoptosis in mammalian organisms; however, the expression levels of CLOCK in varicose veins remain to be elucidated. The aim of the present study was to detect the expression of the circadian clock gene in initial and advanced varicose lesions and analyze the correlation between the CLOCK gene, HIF-1 $\alpha$, and its target gene, vascular endothelial growth factor (VEGF), in VVs. Sections of the great saphenous veins (GSVs) were obtained from patients undergoing ligation and stripping for $\mathrm{VVs}(\mathrm{n}=70)$ and a control group undergoing coronary artery bypass grafting with GSV harvest $(n=11)$. All VV patients had incompetent GSVs, according to color flow duplex scanning. C-class VVs were determined according to the clinical-etiology-anatomy-pathophysiology classification for venous diseases following physical examination of the patients with VV. Reverse transcription-quantitative polymerase chain reaction was used to determine the expression levels of the CLOCK gene, HIF-1 $\alpha$ and VEGF. Immunohistochemical analysis was also performed. The patients with VVs were divided into those with initial varicose lesions (C3 and $\mathrm{C} 4$ ) and advanced varicose lesions (C5 and C6). In total, 21 of the patients had $\mathrm{C} 3$ lesions, 23 had $\mathrm{C} 4$ lesions, 14 had $\mathrm{C} 5$ lesions and 12 had C6 chronic venous disease. The expression of the
\end{abstract}

Correspondence to: Professor Longhua Fan, Department of Vascular Surgery, Qingpu Branch of Zhongshan Hospital, Fudan University, 1158 Park Road, Qingpu, Shanghai 200032, P.R. China

E-mail: fan.longhua@zs-hospital.sh.cn

${ }^{*}$ Contributed equally

Key words: varicose veins, clock, hypoxia-indicible factor-1 $\alpha$, vascular endothelial growth factor
CLOCK gene was significantly higher in the VV lesions of the GSV, compared with the normal GSVs $(\mathrm{P}<0.0001)$. The same trend was found in the expression levels of HIF-1 $\alpha$ and its target gene, VEGF, in the VV lesions ( $\mathrm{P}=0.003$ and $\mathrm{P}<0.0001$, respectively). Subgroup analysis revealed that the expression levels of the CLOCK gene, HIF- $1 \alpha$ and VEGF were significantly higher in the advanced stage varicose lesions, compared with the initial varicose lesions $(\mathrm{P}<0.0001, \mathrm{P}=0.0014$ and $\mathrm{P}<0.0001$, respectively). However, no statistically significant difference was identified in the expression levels of the aforementioned genes in the $\mathrm{C} 3$ and $\mathrm{C} 4$ lesions. The results demonstrated that the expression gene levels of CLOCK, HIF-1 $\alpha$ and its target gene, VEGF, increased significantly in advanced stage varicose lesions. Therefore, upregulation of the CLOCK gene in the vessel walls of veins may be involved in the pathogenesis of VVs and the progression of venous disease.

\section{Introduction}

Varicose veins (VVs) are a common pathology affecting the lower extremities and are manifested by a range of conditions, including pain, ankle edema, itch, eczema, lipodermatosclerosis and ulceration, affect approximately one third of the adult western population and $20 \%$ of the adult Chinese population (1). Despite several hypotheses, the mechanism involved in VVs remains to be elucidated. Increasing lines of evidence indicates that venous wall alterations and dilation are the primary events responsible for the formation of VVs and suggests that chronic venous insufficiency, characterized by symptoms or signs produced by venous hypertension, may be the result of venous wall changes. Structural and biochemical changes, which have been reported in the walls of VVs include intimal hyperplasia, dysregulated apoptosis, smooth muscle cell proliferation, abnormal glucose metabolism, altered angiogenesis, changes in collagen and elastin content, and imbalances of matrix metalloproteases and their tissue inhibitors $(1,2)$.

Venous hypoxia secondary to blood stasis in chronic venous disease has been hypothesized to contribute to changes in the walls of VVs (3). Hypoxia-inducible factors (HIFs) are nuclear transcription factors. HIF- $\alpha$ dimerizes with HIF- $\beta$ in 
the nucleus and binds the hypoxia-responsive element of target genes, which regulate their transcription responses to altered oxygenation $(4,5)$. In vitro studies have clearly demonstrated that hypoxia upregulates the formation of new vessels through activation of the HIF pathway, leading to the release of pro-angiogenic factors, including vascular endothelial growth factor (VEGF) and basic fibroblast growth factor $(6,7)$. Together with nitric oxide, VEGF, one of the target genes of HIF-1, is central in maintaining vascular integrity and reactivity by mediating vaso-permeability and dilatory responses $(8,9)$.

In mammals, several behavioral and physiological processes exhibit circadian ( $24 \mathrm{~h}$ ) rhythms, which are controlled by a clock system. This system includes a central circadian clock residing in the hypothalamic suprachiasmatic nucleus (SCN) and a peripheral clock located in a number of peripheral tissues (10). Previous studies have demonstrated that peripheral tissues and cells contain a similar clock system to that in the SCN $(11,12)$. It has been reported that the circadian rhythmicity of human plaque-derived vascular smooth muscle cells (VSMCs) differs from that of normal carotid VSMCs (13). Rhythmic changes in the expression of circadian clock genes, including circadian locomotor output cycles kaput (CLOCK), in plaque-derived VSMCs may be involved in the process of vessel disease. This hypothesis is supported by increasing evidence that cross-talk between mediators of hypoxic and circadian pathways can regulate target genes (14). To further understand the pathology of VVs, the present study compared the expression of the CLOCK gene in initial and advanced varicose lesions, and examined the correlation between the CLOCK gene, HIF-1 $\alpha$ and its target gene, VEGF, in VVs.

\section{Patients and methods}

Patient recruitment. The procedures performed in the present study were performed following the principles outlined in the Declaration of Helsinki and approved by the Ethics Committee of Zhongshan Hospital, Fudan University (Shanghai, China). All human tissues were collected following the provision of informed consent by the patients. Following removal of adherent connective tissue, all venous specimens were snap-frozen in liquid nitrogen (Shanghai Pujiang Special Gas Co., Ltd., Shanghai, China) and then stored at $-80^{\circ} \mathrm{C}$ or preserved in $4 \%$ paraformaldehyde (Sangon Biotech Co., Ltd., Shanghai, China) until use.

The samples of VVs were obtained from symptomatic patients undergoing saphenofemoral ligation and stripping of the great saphenous vein (GSV) for primary VVs due to incompetence of the GSV. Prior to surgery, a duplex ultrasound scan was performed in a vascular laboratory by a qualified independent vascular surgeon. Prior to removal of the varicose lesions, physical examination and hemodynamic findings were used to classify the stage of disease, according to the clinical-etiology-anatomy-pathophysiology (CEAP) criteria of the American Venous Forum for chronic lower-extremity venous disease (15). As venous ulcers in C5 patients (a healed ulcer) or C6 patients (an active ulcer) can significantly reduce health-associated quality of life, the patients with VV were divided into those with initial varicose lesions (C3 and $\mathrm{C} 4)$ and advanced varicose lesions (C5 and C6).
Samples of a normal control vein were obtained from patients undergoing cardiac bypass procedures, who had no symptoms or clinically evident signs of varicose disease in either limb. The veins were confirmed with a duplex ultrasound scan using a Philips iU22 (Philips, Bothell, WA, USA) ultrasound system, which was performed in the vascular laboratory at the Qingpu Branch of Zhongshan Hospital by a qualified independent vascular surgeon. The veins were confirmed to be normal with a preoperative duplex scan, which indicated that the vessel was disease-free and without valvular incompetence in any segment. Patients with a history of recent infection, rheumatoid arthritis, steroid use, cancer or connective tissue disease, those $<18$ years of age or those who did not sign a consent form, were excluded from the investigation.

RNA isolation and reverse-transcription quantitative polymerase chain reaction ( $R T-q P C R)$. Total RNA from each vein was extracted using TRIzol reagent (Invitrogen Life Technologies, Carlsbad, CA, USA) in an RNase-free environment, according to the manufacturer's standard instructions. First-strand cDNA was synthesized and amplified from $1 \mu \mathrm{g}$ of the total RNA using the ReverTra Ace qPCR RT kit (Toyobo Co., Ltd., Osaka, Japan). Messenger RNA (mRNA) levels of the target genes were determined using an ABI 7900HT machine (Applied Biosystems Life Technologies, Foster City, CA, USA) in triplicate, in $10 \mu 1$ reaction mixtures containing SYBR Green Real-time PCR master mix (Bio-Rad Laboratories, Inc., Hercules, CA, USA). The relative quantification of gene expression was analyzed from the measured threshold cycles $(\mathrm{Ct})$ using the $2^{-\Delta \Delta \mathrm{Ct}}$ method, as described previously (16). Glyceraldehyde-3-phosphate dehydrogenase (GAPDH) was used as an internal standard to normalize the expression level of other genes. Primers were designed by PRIMER 5.0 (Premier Biosoft International, Palo Alto, CA, USA) and synthesized by Sangon Biotech Co., Ltd., as follows: CLOCK, forward 5'-GGCATGTCCCAGTTTCAGTTT-3' and reverse 5'-ATGCGTGTCCGTTGTTCCAAT-3'; HIF-1 $\alpha$, forward 5'-CACCACAGGACAGTACAGGAT-3' and reverse 5'-CGTGCTGAATAATACCACTCACA-3'; VEGF, forward 5'-AGGGCAGAATCATCACGAAGT-3' and reverse 5'-AGG GTCTCGATTGGATGGCA-3' and GAPDH, forward 5'-TGT TGCCATCAATGACCCCTT-3' and reverse 5'-CTCCACGAC GTACTCAGCG-3'.

Immunohistochemistry (IHC). For histological analysis of the venous tissue, $4 \mu \mathrm{m}$ sections of the paraffin-embedded veins were used. Hematoxylin and eosin staining was used for general histological evaluation, and calcification was observed using von Kossa staining (cat. no. ab1506871; Abcam, Cambridge, MA, USA). Formalin-fixed paraffin embedded sections ( $4 \mu \mathrm{m}$ thickness) of varicose veins were used for CLOCK immunostaining, according to the manufacturer's standard instructions, as described previously (17). In brief, the slides were deparaffinized in xylene and rehydrated with alcohol prior to being placed in a $3 \% \mathrm{H}_{2} \mathrm{O}_{2} /$ methanol blocking solution to quench endogenous peroxidase activity. This was followed by subsequent antigen retrieval. The non-specific binding was blocked with normal horse or goat serum. The slides were incubated with rabbit anti-human polyclonal antibody for 10 minutes at $95^{\circ} \mathrm{C}$ in the specified 
Table I. Patient demographics and clinical risk factors.

\begin{tabular}{lcccr}
\hline & \multicolumn{2}{c}{ Varicose vein group } & & \\
\cline { 2 - 3 } Characteristic & Initial $(\mathrm{n}=44)$ & Advanced $(\mathrm{n}=26)$ & Control group $(\mathrm{n}=11)$ & P-value \\
\hline CEAP classification & $\mathrm{C} 3$ and C4 & C5 and C6 & Normal & - \\
Age (years) & $58.6 \pm 9.2$ & $57.0 \pm 7.1$ & $67.4 \pm 6.9^{\text {a }}$ & $\mathrm{P}<0.05$ \\
Male:female & $25: 19$ & $19: 7$ & $9: 2$ & $\mathrm{P}=0.181$ \\
Hypertension (n) & 14 & 9 & $8^{\mathrm{b}}$ & $\mathrm{P}=0.040$ \\
Diabetes mellitus (n) & 4 & 2 & $7^{\mathrm{c}}$ & $\mathrm{P}<0.001$ \\
Dyslipidemia (n) & 9 & 10 & 5 & $\mathrm{P}=0.131$ \\
COPD (n) & 0 & 0 & 1 & $\mathrm{P}=0.136$ \\
\hline
\end{tabular}

Results are presented as the mean \pm standard deviation. Statistical analyses of the groups were performed using Student's t-test. A one-way analysis of variance, followed by the Fisher's exact test, was performed when appropriate. ${ }^{\mathrm{a}} \mathrm{P}=0.040$, vs. varicose vein group; ${ }^{\mathrm{b}} \mathrm{P}<0.001$, vs. varicose vein group; ${ }^{\mathrm{C}} \mathrm{P}<0.001$, vs. varicose vein group. COPD, chronic obstructive pulmonary disease; CEAP, clinical-etiology-anatomy-pathophysiology.

antigen retrieval solution ( $\mathrm{pH} 6.0$, Abcam) at a 1:50 dilution overnight in a humidified chamber at $4^{\circ} \mathrm{C}$. Following washing with phosphate-buffered saline, the slides were incubated with goat anti-rabbit IgG conjugated to a horseradish peroxidase-labeled polymer (Envision System; Dako, Carpinteria, CA, USA) for $30 \mathrm{~min}$ at room temperature. Reactions were performed using 3,3'-diaminobenzidine chromogen and counterstained with hematoxylin. Negative controls comprised of tissue sections incubated with non-specific $\operatorname{IgG}$ in place of the primary antibody.

All sections were quantitatively analyzed and scored by two experienced pathologists under a light microscope. The scoring of the IHC was based on two independent criteria: The proportion of immunopositive cells and their intensity of immunoreactivity. The percentage of immunopositive cells was categorized as follows: $0,<10 \% ; 1, \geq 10$ to $<25 \%$; 2,25 to $<50 \% ; 3, \geq 50$ to $<75 \%$ and $4, \geq 75 \%$. The intensity of staining was categorized by the relative intensity as follows: 0 , no positivity; 1 , weak positivity; 2 , moderate positivity and 3, marked positivity. A final immunoreactivity score of each section was assigned by multiplying the two individual scores.

Statistical analysis. Statistical analysis was performed using SPSS software, version 19.0 (IBM SPSS, Armonk, NY, USA). The results are expressed as the mean \pm standard deviation. The values for mRNA levels are presented as relative values in all experiments. Student's t-test was performed to examine the differences between the groups. A one-way analysis of variance, followed by Fisher's test, was used when appropriate. Pearson's correlation analysis was performed to examine the correlation between CLOCK and HIF-1 $\alpha$ or the levels of VEGF in the human VVs. $\mathrm{P}<0.05$ was considered to indicate a statistically significant difference.

\section{Results}

Patient demographics and clinical characteristics. The demographics and characteristics of the patients in the clinical investigations are summarized in Table I. The patients with
VVs exhibited incompetence of the saphenofemoral junction, combined with superficial and perforator reflux (CEAP classification, C3-6). The mean age of the control group was 67.4 years (standard deviation, 6.9) and the ratio of males to females was 9:2. The patients with either initial VVs or advanced VVs were significantly younger than the control patients $(58.6 \pm 9.2$ and 57.0 \pm 7.1 years, vs. 67.4 \pm 6.9 years, respectively; $\mathrm{P}<0.05)$. Fewer patients in the $\mathrm{VV}$ group had hypertension or diabetes mellitus, compared with the control group ( $\mathrm{P}=0.040$ and $\mathrm{P}<0.001$, respectively). No other differences in the demographics were identified among the three groups.

Quantification of the gene expression of CLOCK in VVs and normal vein tissues. To analyze the gene expression of CLOCK in the veins, RT-qPCR analysis of the veins from VVs and normal control veins was performed. As shown in Fig. 1A, a high mRNA expression level of CLOCK was detected in the VVs. Densitometric analysis revealed that the mRNA levels of CLOCK in the VVs were higher than those in the normal veins (control group). Although variations between individuals were observed, the expression pattern was similar in all the vein samples examined. The present study subsequently investigated whether the deterioration in the VVs accounted for the elevated gene expression of CLOCK in the VVs. Subgroup comparison of the C3 and C4 lesions revealed no significant differences in the gene expression of CLOCK in these initial VVs (Fig. 1B). However, comparison of the mRNA expression level of CLOCK in the advanced varicose lesions (C5 and C6), with those in the initial varicose lesions ( $\mathrm{C} 3$ and $\mathrm{C} 4)$ revealed significantly higher levels in the $\mathrm{C} 5$ and $\mathrm{C} 6$ lesions (Fig. 1C). To confirm these results, IHC analysis was performed to investigate the expression of CLOCK in the VVs and normal control veins. The IHC analysis revealed that the expression of CLOCK was predominantly located in the cytoplasm (Fig. 2). Normal control veins exhibited negative or weak staining, while staining in the VVs was often more marked (Fig. 2). Taken together, these data revealed that upregulation in the mRNA expression of CLOCK is a characteristic of VVs. 
A

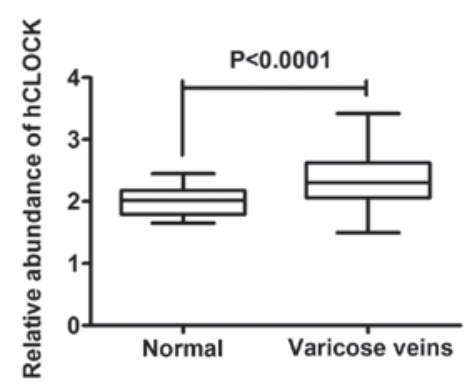

B

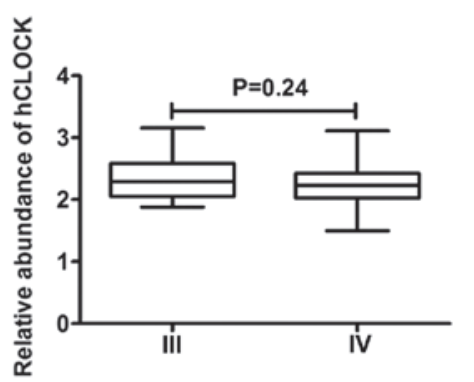

C

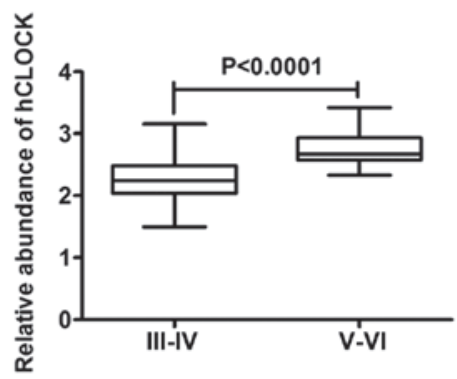

Figure 1. Difference in the relative expression of CLOCK in varicose veins sample tissues. Comparison of the differences in the expression levels of CLOCK between (A) normal veins tissues and varicose vein tissues; (B) C3 (III) subgroup and C4 (IV) subgroup tissues; (C) C3-C4 (III-IV) and C5-C6 (V-VI) subgroup. Expression levels of CLOCK were determined using reverse-transcription-quantitative polymerase chain reaction and normalized against an endogenous control (GAPDH). Data are expressed as the mean \pm standard deviation. CLOCK, circadian locomotor output cycles kaput.

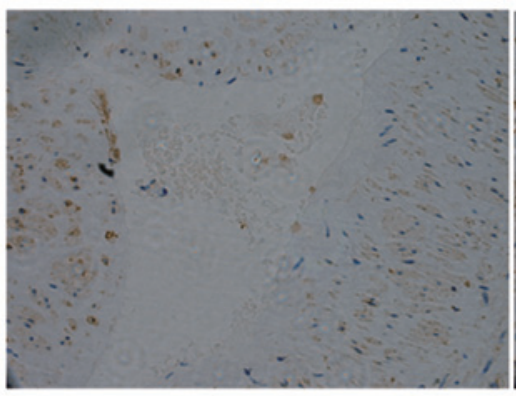

Normal

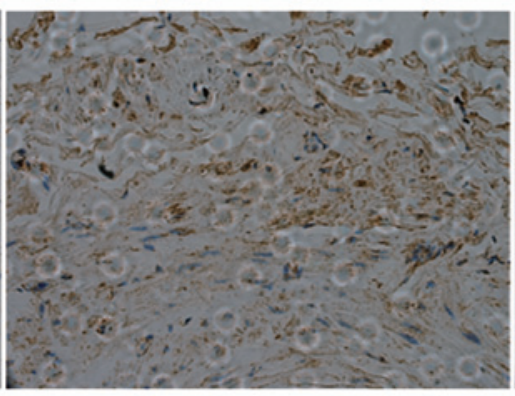

Initial VV

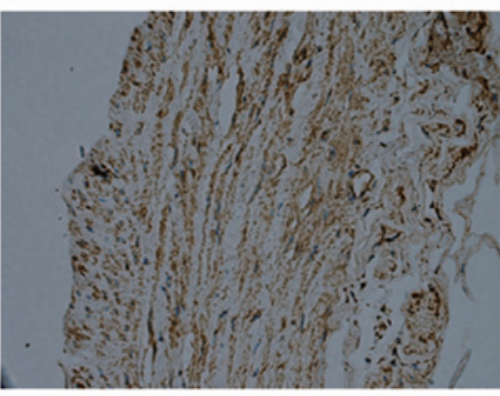

Advanced VV

Figure 2. Detection of the expression of CLOCK using immunohistochemistry. Representative photomicrographs of the expression of CLOCK in normal veins (left), which was commonly weak or absent; C3-C4 (middle) veins, which was commonly weak or moderate and C5-C6 (right), which commonly exhibited more intense immunostaining of CLOCK (original magnification, $\mathrm{x} 400$ ). VV, varicose vein; CLOCK, circadian locomotor output cycles kaput.

A

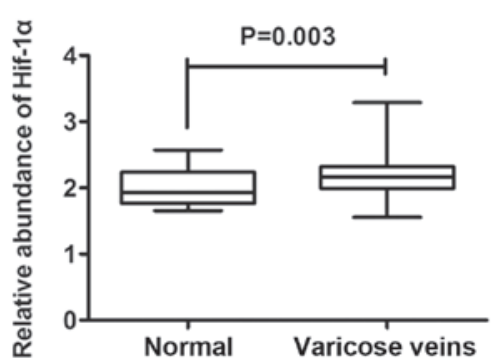

B

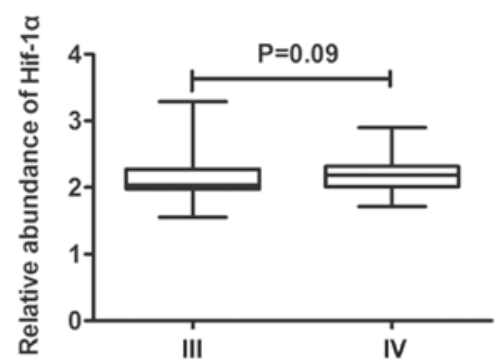

C

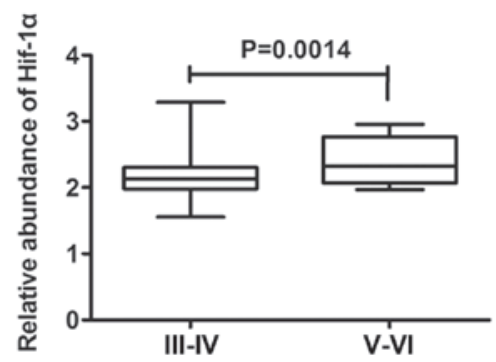

Figure 3. Differences in the relative expression of HIF-1 $\alpha$ in varicose vein sample tissues. Reverse transcription-quantitative polymerase chain reaction analysis of the expression of HIF-1 $\alpha$ between (A) normal veins tissues and varicose vein tissues; (B) C3 and C4 subgroup (III and IV) tissues; and (C) C3-4 (III-IV) and C5-6 subgroup (V-VI). Data are expressed as the mean \pm standard deviation. HIF, hypoxia-inducible factor.

A

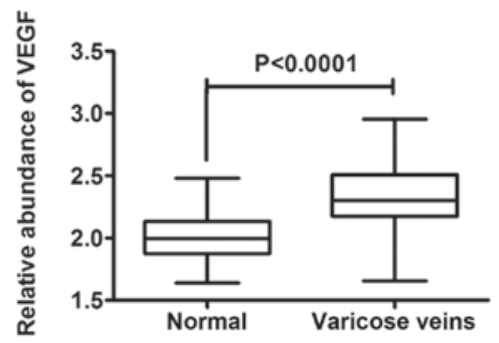

$\mathbf{B}$

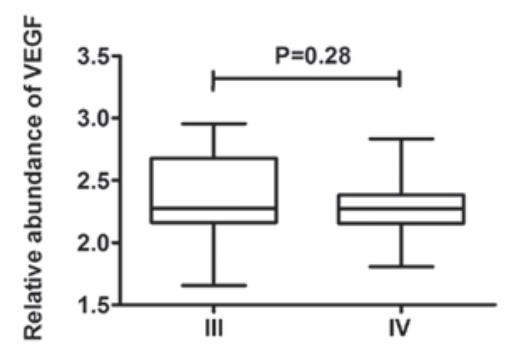

C

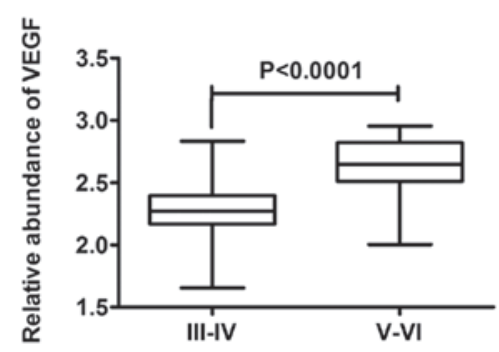

Figure 4. Differences in the relative expression of VEGF in varicose vein sample tissues. Relative expression levels of VEGF between (A) normal veins tissues and varicose vein tissues; (B) C3 and C4 subgroup tissues (III-IV) and (C) C3-4 (III-IV) and C5-6 (V-VI) subgroup. Data are expressed as the mean \pm standard deviation. VEGF, vascular endothelial growth factor. 
A

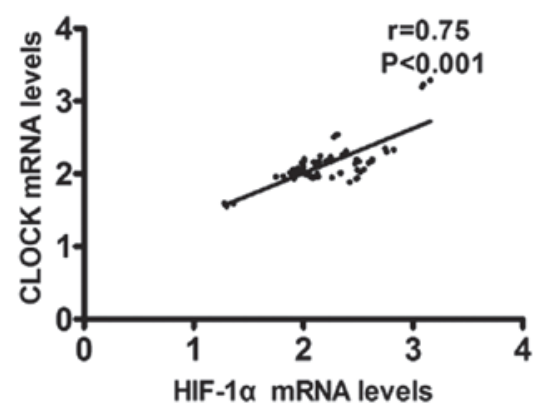

B

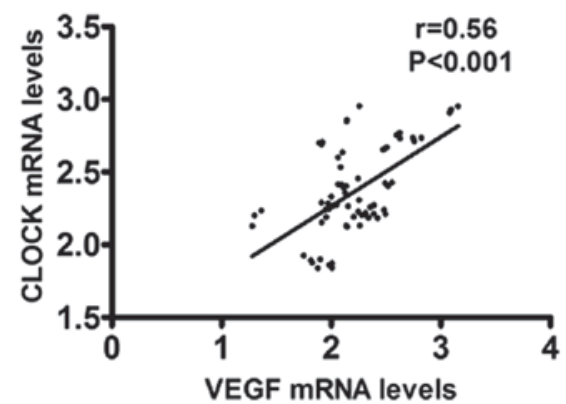

Figure 5. Correlation between the expression levels of CLOCK, HIF-1 $\alpha$ and VEGF in varicose vein sample tissues. (A) Correlation between the expression levels of CLOCK and HIF-1 $\alpha$ in varicose vein sample tissues, determined using Pearson's product-moment correlation coefficient. (B) Correlation between the expression levels of CLOCK and VEGF in varicose vein sample tissues, determined using Pearson's product-moment correlation coefficient. VEGF, vascular endothelial growth factor; HIF, hypoxia-inducible factor. CLOCK, circadian locomotor output cycles kaput.

Expression levels of HIF-1 $\alpha$ and VEGF are elevated in $V V s$. Previous studies have demonstrated that hypoxia increases the protein expression levles of CLOCK in mice, and the co-operative effect of HIF- $1 \alpha$ and CLOCK lead to the transcriptional activity of vasopressin $(14,18)$. In the present study, the expression levels of HIF-1 $\alpha$ and VEGF were assessed. Compared with the control veins, RT-qPCR revealed a significant increase in the mRNA expression levels of HIF-1 $\alpha$ in the VVs (Fig. 3A), however, no significant differences were observed between the $\mathrm{C} 3$ and $\mathrm{C} 4$ subgroups (Fig. 3B). Furthermore, it was also observed that the mRNA expression levels of HIF-1 $\alpha$ were significantly increased in the advanced varicose lesions (C5 and C6), compared with those in the initial varicose lesions (C3 and C4; Fig. 3C). Similarly, RT-qPCR revealed a significant increase in the mRNA expression levels of VEGF in the VVs, compared with the normal control veins (Fig. 4A), however, no significant differences were observed between the $\mathrm{C} 3$ subgroup and C4 subgroup (Fig. 4B). In addition, the mRNA expression levels of VEGF were significantly increased in the advanced varicose lesions (C5 and C6), compared with those in the initial varicose lesions (C3 and C4; Fig. 4C). Despite individual variations, the same expression pattern was observed in all the specimens assessed. Together, these results revealed marked expression levels of HIF-1 $\alpha$ and VEGF associated with venous disease, the expression pattern of which were distinct, compared with those observed in the control veins.

Upregulation of CLOCK is associated with the expression of $H I F-1 \alpha$ and VEGF in VVs. To better understand the clinical significance of CLOCK in VVs, the association between the expression of CLOCK, HIF- $1 \alpha$ and VEGF in VVs was investigated. A relatively higher expression level of CLOCK was detected in the early and advanced VV tissues, with high expression levels of HIF-1 $\alpha$. The expression levels of CLOCK and HIF-1 $\alpha$ were positively correlated in the early and advanced VV tissues ( $\mathrm{r}=0.75 ; \mathrm{P}<0.001$; Fig. $5 \mathrm{~A})$. The same trends were observed in the expression levels of CLOCK and VEGF in the advanced VVs $(r=0.56, \mathrm{P}<0.001$; Fig. 5B).

\section{Discussion}

At present, the pathogenesis of VVs remains to be fully elucidated. Understanding the pathophysiology of chronic venous disease is important to establish pharmacological approaches to complement current treatment strategies. For the first time, to the best of our knowledge, the present study demonstrated that high mRNA expression of CLOCK is a hallmark of VVs, and that upregulation in the expression of the CLOCK gene is correlated with HIF-1 $\alpha$ following deterioration in VVs. These findings indicate a potential link between the expression of HIF-1 $\alpha$ and the circadian clock in the pathogenesis of this disease.

Venous hypoxia has been hypothesized to contribute to $\mathrm{VV}$ wall changes and it is well known that activation of the HIF pathway is increased in cells and tissues associated with hypoxia (19). In the present study, the expression of HIF-1 $\alpha$ in the VVs was elevated, compared with that of the control group containing normal veins, the results of which are in agreement with previous in vitro studies $(20,21)$. The increased expression of HIF in the VVs is a significant finding, as HIFs regulate $\sim 1 \%$ of human genes (5). Several HIF target genes are involved in functions, which are known to cause pathophysiological changes in the walls of veins. However, what stage of VV formation the HIF pathway is activated remains to be elucidated. The present study demonstrated for the first time, to the best of our knowledge, that the pathway was activated following the deterioration of VVs (in C5 and C6 lesions). Analysis of the subgroups revealed that the expression of HIF-1 $\alpha$ in the advanced varicose lesions was significantly higher, compared with that of the initial varicose lesions.

Several groups have demonstrated that hypoxia can stimulate the production of VEGF $(8,22)$. VEGF is central in the maintenance of vascular integrity and reactivity by mediating vaso-permeability and dilatory responses (9). VEGF, which is produced in vascular smooth muscle, can stimulate the production of nitric oxide from the vascular endothelium, and continued and elevated expression of VEGF then leads to elevated production of nitric oxide, resulting in the generation of reactive oxygen species, contributing to the further development of the pathology (8). Although it has been previously demonstrated that patients with primary $\mathrm{VV}$ exhibit a loss of VEGF release following mild, experimentally-induced venous stasis that has been observed in control individuals (23), the present study clearly demonstrated a significant rise in the expression of VEGF in the VVs, as well as altered expression of VEGF following deterioration 
in the veins. The ability to maintain or regulate the levels of VEGF may be reduced in the VVs.

Notably, our previously study demonstrated that VEGF $\alpha$ is one of a few genes expressed in a circadian rhythm-dependent manner in the hearts of C57BL/6J and $\mathrm{ApoE}^{-/-}$mice (24). In mammals, several behavioral and physiological processes exhibit circadian $(\sim 24 \mathrm{~h})$ rhythms, which are controlled by a clock system. The circadian rhythmicity of peripheral tissues is considered to be uniquely controlled by the SCN via neural and humoral signals. Previous studies have demonstrated that peripheral tissues and cells contain a similar clock system to that in the $\operatorname{SCN}(11,12)$. Our previous study revealed that the levels and rhythms of the expression of core circadian genes were altered in human carotid plaque-derived VSMCs (13). Therefore, in the present study, focus was placed on the potential role of the CLOCK gene in the pathogenesis of varicose lesions. The present study revealed, for the first time, to the best of our knowledge, that the expression of the CLOCK gene was significantly upregulated in the advanced VVs, compared with normal control veins or initial VVs. This finding further supports the hypothesis that changes in the expression of circadian clock genes may be involved in the progression of vascular diseases.

In common with several features of VVs, the cause and effect association between the CLOCK gene and VVs may be difficult to ascertain, particularly whether the association contributes to a vicious cycle of disease progression. The control of gene transcription and the maintenance of vascular reactivity may be altered by a causative mechanism or simply by the pathological process. As noted previously, increasing evidence has demonstrated that cross-talk between mediators of the hypoxic and circadian pathways regulate target genes. At the molecular level, transient transfection experiments investigating the effect of hypoxia on the circadian clock have revealed that the CLOCK gene interacts with HIF-1 $\alpha$ and drives transcription (14). HIF-1 $\alpha$ does not form a DNA binding complex with the CLOCK gene at E-box A, the control element of several circadian clock genes and the core sequence of the hypoxia-responsive element. However, the brain and muscle ARNT-like protein 1 complex at E-box A is regulated by co-transfected HIF-1 $\alpha /$ CLOCK. Therefore, the E-box may be a key element in the interaction between hypoxia signaling and the circadian clock (14). A previous study revealed a bidirectional link between the hypoxic and circadian pathway (25). Further examination of such genes may assist in elucidating the mechanism underlying the development of VVs.

In conclusion, the present study demonstrated a significant increase in the gene expression of CLOCK, as well as HIF-1 $\alpha$ and its target gene, VEGF, in advanced varicose lesions. The upregulation of the circadian clock gene in venous vessels may be involved in the pathogenesis of VVs and promote the progression of venous disease. However, further investigations are required to ascertain the correlations among the CLOCK gene, hypoxia and HIF- $1 \alpha$, and the bidirectional link between the hypoxic and circadian pathways in the pathogenesis of VVs. Investigations are also required to detect the protein expression of these circadian clock genes and identify other downstream genes that they mediate. Pharmacological therapy aimed at hypoxia signaling and the circadian clock may have a role in the management of venous diseases in the future.

\section{Acknowledgements}

The present study was supported a by grant from the Zhongshan Hospital Youth Talent Training Program (grant. no. 201514).

\section{References}

1. Lim CS and Davies AH: Pathogenesis of primary varicose veins. Br J Surg 96: 1231-1242, 2009.

2. Raffetto JD and Khalil RA: Mechanisms of varicose vein formation: Valve dysfunction and wall dilation. Phlebology 23: 85-98, 2008.

3. Lim CS, Gohel MS, Shepherd AC, Paleolog E and Davies AH: Venous hypoxia: A poorly studied etiological factor of varicose veins. J Vasc Res 48: 185-194, 2011.

4. Semenza GL: Regulation of oxygen homeostasis by hypoxia-inducible factor 1. Physiology (Bethesda) 24 97-106, 2009.

5. Muz B, Khan MN, Kiriakidis S and Paleolog EM: Hypoxia. The role of hypoxia and HIF-dependent signalling events in rheumatoid arthritis. Arthritis Res Ther 11: 201, 2009.

6. Calvani M, Rapisarda A, Uranchimeg B, Shoemaker RH and Melillo G: Hypoxic induction of an HIF-1alpha-dependent bFGF autocrine loop drives angiogenesis in human endothelial cells. Blood 107: 2705-2712, 2006.

7. Zhu XY, Daghini E, Chade AR, Lavi R, Napoli C, Lerman A and Lerman LO: Disparate effects of simvastatin on angiogenesis during hypoxia and inflammation. Life Sci 83: 801-809, 2008.

8. Servos S, Zachary I and Martin JF: VEGF modulates NO production: The basis of a cytoprotective effect? Cardiovasc Res 41: 509-510, 1999.

9. Tomasian D, Keaney JF and Vita JA: Antioxidants and the bioactivity of endothelium-derived nitric oxide. Cardiovasc Res 47: 426-435, 2000.

10. Moore RY: Circadian rhythms: Basic neurobiology and clinical applications. Annu Rev Med 48: 253-266, 1997.

11. Balsalobre A: Clock genes in mammalian peripheral tissues. Cell Tissue Res 309: 193-199, 2002.

12. Singh D, Rani S and Kumar V: Daily expression of six clock genes in central and peripheral tissues of a night-migratory songbird: Evidence for tissue-specific circadian timing. Chronobiol Int 30: 1208-1217, 2013.

13. Lin C, Tang X, Zhu Z, Liao X, Zhao R, Fu W, Chen B, Jiang J, Qian R and Guo D: The rhythmic expression of clock genes attenuated in human plaque-derived vascular smooth muscle cells. Lipids Health Dis 13: 14, 2014.

14. Ghorbel MT, Coulson JM and Murphy D: Cross-talk between hypoxic and circadian pathways: Cooperative roles for hypoxia-inducible factor 1alpha and CLOCK in transcriptional activation of the vasopressin gene. Mol Cell Neurosci 22: 396-404, 2003.

15. Classification and grading of chronic venous disease in the lower limbs. A consensus statement. Ad Hoc Committee, American Venous Forum. J Cardiovasc Surg (Torino) 38: 437-441, 1997.

16. Zhu XC, Dong QZ and Zhang XF, et al.: microRNA-29a suppresses cell proliferation by targeting SPARC in hepatocellular carcinoma. Int J Mol Med 30: 1321-1326, 2012

17. Hashimoto T, Yanaihara N, Okamoto A, Nikaido T, Saito M, Takakura S, Yasuda M, Sasaki H, Ochiai K and Tanaka T: Cyclin D1 predicts the prognosis of advanced serous ovarian cancer. Exp Ther Med 2: 213-219, 2011.

18. Chilov D, Hofer T, Bauer C, Wenger RH and Gassmann M: Hypoxia affects expression of circadian genes PER1 and CLOCK in mouse brain. FASEB J 15: 2613-2622, 2001.

19. Semenza GL: Defining the role of hypoxia-inducible factor 1 in cancer biology and therapeutics. Oncogene 29: 625-634, 2010.

20. Lim CS, Kiriakidis S, Paleolog EM and Davies AH: Increased activation of the hypoxia-inducible factor pathway in varicose veins. J Vasc Surg 55: 1427-1439, 2012. 
21. Lee JD, Yang WK and Lee TH: Increased expression of hypoxia-inducible factor-1alpha and $\mathrm{Bcl}-2$ in varicocele and varicose veins. Ann Vasc Surg 26: 1100-1105, 2012

22. Thomas KA: Vascular endothelial growth factor, a potent and selective angiogenic agent. J Biol Chem 271: 603-606, 1996.

23. Hollingsworth SJ, Tang CB, Dialynas M and Barker SG: Varicose veins: Loss of release of vascular endothelial growth factor and reduced plasma nitric oxide. Eur J Vasc Endovasc Surg 22: 551-556, 2001.
24. Xu C, Lu C and Hua L, Jin H, Yin L, Chen S and Qian R: Rhythm changes of clock genes, apoptosis-related genes and atherosclerosis-related genes in apolipoprotein E knockout mice. Can J Cardiol 25: 473-479, 2009.

25. Egg M, Köblitz L, Hirayama J, Schwerte T, Folterbauer C, Kurz A, Fiechtner B, Möst M, Salvenmoser W, Sassone-Corsi P, et al: Linking oxygen to time: The bidirectional interaction between the hypoxic signaling pathway and the circadian clock. Chronobiol Int 30: 510-529, 2013. 